Obesity in Toddlers and Young Children: Causes and

\title{
Consequences
}

\section{Atul Singhal}

From: ${ }^{1}$ The Childhood Nutrition Research Centre; Population, Policy and

Practice Programme, UCL Great Ormond Street Institute of Child Health 30 Guilford Street, London WC1N 1EH

Address for correspondence: Professor A Singhal Tel: 442070952389

E mail: a.singhal@ich.ucl.ac.uk

Short title: Obesity in young children 


\section{Abstract}

The rapid rise in obesity in toddlers and young children (aged 0-5 years) is a major concern for public health globally. Understanding risk factors for obesity in the early years is therefore fundamental to help guide parents, educators, and health care professionals caring for young children and to develop preventative strategies. Most research has focused on biological risk factors, which can be broadly categorised as genetic predisposition, poor diet (and the behaviours that influence excessive food intake), insufficient physical activity, and the role of developmental factors in early life that influence long-term health. The latter includes establishment of dietary habits and dietary patterns in young (preschool) children and the impact of a high protein intake in increasing the risk of later obesity. Other risk factors particularly relevant to young children included inadequate sleep, high consumption of sugar-sweetened drinks and large food portions. Understanding the causes of obesity in preschool children is particularly important in view of long-term detrimental consequences of obesity in this age group on the risk of obesity and cardio-metabolic disease in adults. The present chapter reviews causes of obesity in preschool children and its consequences for long-term health, focusing particularly on modifiable nutritional risk factors.

\section{Introduction}


The huge increase in prevalence of obesity over the last 40 years in nearly all 200 countries studied is a major challenge for health care systems worldwide [1]. According to the World Health Organisation (WHO), in 2016 $39 \%$ of adults globally were overweight and $13 \%$ obese [2]. Importantly this problem is not confined to adults and high-income countries [1]. In 2016, for instance, over 41 million children under the age of 5 years were overweight or obese, nearly half of whom lived in Asia [2]. Despite this growing threat to the health of populations, few treatments for obesity are available [3], especially those affordable or suitable for low- and middleincome countries. Once established, obesity is difficult to reverse and most interventions for obesity are expensive, difficult to implement at scale, and show disappointing long-term results [3]. Hence, prevention of obesity is critical, particularly in the early years where there may be an opportunity to affect the long-term trajectories of weight gain and have greater sustained effects $[4,5]$.

Several recent reports have highlighted the importance of preventing obesity in the early years. These include the WHO's report on ending childhood obesity [4] and the UK Chief Medical Officer's report, 'Time to Solve Childhood Obesity' [5]. Both reports emphasise the critical role of the first few years in establishing good nutritional and physical activity behaviours that can help prevent long-term excessive weight gain. This concept is strongly supported by data showing that, in richer countries at least, most excess weight at adolescence is gained before 5 years of age 
$[6,7]$. The present chapter reviews the possible causes of obesity in young children and its consequences for long-term health, focusing particularly on modifiable nutritional risk factors.

\section{Environmental Risk Factors for Obesity}

At the simplest level, obesity results from an imbalance between energy intake and energy expenditure. However childhood obesity cannot be due to an energy imbalance as result of voluntary lifestyle choices, particularly in preschool children. The factors that predispose to a positive energy balance are complex and include genetic predisposition and exposure to an unhealthy 'obesogenic' environment such as the easy availability and affordability of highly palatable, energy-dense foods $[4,5]$. Social determinants of health (the conditions in which people are born grow, work and live such as the physical environment) also have a major impact on childhood obesity by, for example, affecting the availability of take-away food outlets and areas for outdoor active play [5]. Commercial determinants of health (e.g. food production, marketing and sale) contribute to the risk of a child becoming overweight. For instance, children respond to TV advertising by consuming more energy dense snacks and foods [8]. However, most research focuses on the biological determinants of obesity, particularly those affecting modifiable risk factors that influence diet and physical activity, but also those affecting the gut microbiome, sleep, psychological responses and appetite. Not only do these biological, social and commercial determinants of health interact to increase the risk 
of obesity, but they also contribute strongly to wide socioeconomic inequalities in this risk. For instance, amongst 4-5 year old children in the UK, obesity in the most deprived $10 \%$ of the population is $6 \%$ greater than the least deprived decile, a disparity that has been increasingly over the last 10 years [5].

Despite the key role of the obesogenic environment, many children living in such an environment do not become obese. Therefore the risk of becoming overweight is highly variable and depends on a child's biological and behavioural response to the environment. A child's biological response to an obesogenic environment is strongly influenced by genetic factors and by 'developmental' or early life factors that 'programme' or influence longterm health [9]. The behavioural response of preschool children in particular, depends on their parent's behaviour which is, in turn, strongly influenced by parental socioeconomic status, knowledge, ethnicity and cultural background. For example, parents may fail to recognise that the child has a weight problem or behaviours such as eating while watching TV may increase the risk of obesity [10]. A lack of knowledge, social or financial resources may also prevent parents from making healthy food choices [11].

Given the myriad of interacting biological, social and commercial determinants of obesity, identifying individual causal factors is both complex and challenging. Hence this review will focus on genetic, dietary 
and developmental factors most commonly linked to childhood obesity and most relevant to children under five.

\section{Genetic Risk Factors}

Children born in families where both parents are obese have $>10$ fold greater risk of becoming obese themselves [12]. Although this increased risk could be either due to genetic mechanisms or by shared familial characteristics such as diet, twin studies and adoption studies (in which identical twins are raised in different families) suggest that $40-70 \%$ of the inter-individual variability of body mass index (BMI) is attributable to genetic factors [13]. Relatively few individual genes (e.g. melanocortin 4 receptor, fat mass and obesity-associated -or FTO gene-, leptin, leptin receptor, and the pro-opiomelanocortin genes) have been identified, many of which lead to severe early onset obesity by influencing the leptinmelanocortin pathway, a regulator of energy intake [13]. More recently, genome-wide association studies have identified $>97$ genetic loci for BMI, with each locus making a small contribution to total risk (the strongest being the FTO gene) [13]. Aggregating these individual genetic variants to provide a genetic risk score shows that individuals with a high polygenic risk score (top $10 \%$ of the population) have a fourfold greater risk of developing obesity than the bottom $10 \%$, an effect that is evident in preschool children [14]. Interesting, the genetic contribution to BMI appears to be greater in children than in adults [15]. 
However, although genes affect an individual's risk of becoming overweight, the huge rise in population obesity over the last 40 years is simply too great and too rapid to be explained predominantly by genetic factors [5]. Observations that intra-individual variance in body weight is similar to inter-individual variance argue against a tight (genetic) control of body weight [16] and the fact that 97 loci for BMI explain only $2.7 \%$ of the variance in population BMI also argues against a dominant role for genetic factors in risk of childhood obesity [13].

\section{Nutritional Risk Factors}

Given their role in energy imbalance, nutritional factors such as energy intake would be expected to be the strongest contributors to obesity in children. However, although diets of preschool children often do not comply with current recommendations [17], there is surprisingly little evidence of an association between diet and risk of obesity, possibly because of limitations in accurately measuring dietary intake in young children [17]. For instance, higher total energy intake is inconsistently associated with obesity (as reviewed [17]), while the macronutrient source of the energy such as total carbohydrate or fat intake also does not affect adiposity [17]. In support of this, a Cochrane systematic review found little consistent evidence of an association between fat intake and body fatness in children [18], while a systematic review of 81 studies found inconclusive associations between dietary factors and 12 obesity related biomarkers (e.g. blood lipids) [19]. In contrast to fat and carbohydrate, protein intake 
in preschool children may affect later (as opposed to current) risk of obesity [20] and is therefore discussed under developmental risk factors below.

Another approach to identifying specific nutritional risk factors for childhood obesity is to consider changes in dietary habits and foods consumed since the onset of the obesity epidemic. Over the last 100 years overall energy intake has fallen alongside the fall in physical activity, but since the 1970 s substantial increases in total energy intake have driven the obesity epidemic and can explain most of the increase in adult bodyweight (as reviewed [5]). The diets of preschool children in the UK have changed markedly and today's children have a far greater intake of sugar and soft drinks, and less vegetables than those of a similar age in the 1950s (a time of post war austerity) [21]. Furthermore, concurrent increases in obesity together with increases in both size of food portions and dietary energy density, suggest these specific nutritional factors could be particularly important in excessive weight gain in young children.

\section{Sugar and Sugar Sweetened Beverages}

The parallel increases in obesity and sugar consumption (particularly sugarsweetened beverages, SSBs) suggests a key role for high sugar foods in development of childhood obesity. Although infants have an innate preference for sweet tastes, there is no nutritional requirement for free sugars (defined as 'all monosaccharides/disaccharides added to foods/beverages, plus sugars naturally present in 
honey/syrups/unsweetened fruit juices and fruit juice concentrates, but not sugar naturally present in intact fruits and lactose naturally present in human and other milks') [22]. Prospective cohort studies and RCTS have consistently shown that high free sugar intake increases the risk of obesity in children and adults (as summarised in systematic reviews from the WHO, UK Scientific Advisory Committee for Nutrition (SACN), and European Society for Paediatric Gastroenterology, Hepatology and Nutrition ESPGHAN- [22]). Recent systematic reviews suggest that this association is most consistent in children aged $<5$ years [23], and that strategies to reduce SSB consumption are effective in this age group [24]. However, few studies have investigated the impact of such interventions on adiposity (probably because of widely recognised difficulties in achieving long-term compliance with dietary interventions). Nonetheless, there is sufficient evidence for public health bodies such as SACN and ESPGHAN to recommend reducing free sugar to less than $5 \%$ of daily energy intake in children aged 2-18 years, and to even less in those under 2 [22]. For an average 3 year old this equates to $13 \mathrm{~g}$ free sugar/day ( $<3$ teaspoons), or an average of $170 \mathrm{ml}$ of fruit juice/day [22]. In practice, this means consuming sugar as part of a main meal and in a natural form such as human milk, milk, unsweetened dairy products, and fresh fruits, and replacing fruit juice with water or unsweetened milk drinks. In addition, as recommended by the WHO [4], several countries (e.g. UK, Mexico, Colombia, Chile, South Africa, France) have successfully applied taxes to reduce SSB consumption. For instance, in the UK, a levy of $24 \mathrm{p} / \mathrm{L}$ on drinks 
containing $8 \mathrm{~g} / 100 \mathrm{ml}$ of sugar, and $18 \mathrm{p} / \mathrm{L}$ on those with $5-8 \mathrm{~g} / 100 \mathrm{ml}$, has reduced the total sugar content of soft drinks sold before (2015) and after the tax (2018) by 30,100 tonnes or $21 \%$ [5].

\section{Portion Size}

Concurrent with the increase in obesity over the last 30 years, there has been a marked increase in the size of food portions given to children [25]. When children and adults are offered larger servings of energy dense foods, overall energy intake increases (the so called 'portion size effect' [25]). Interestingly, in one of the first studies to show this, energy intake increased in children aged 5, but not 3 years, suggesting that younger children have better self-regulation of energy intake [26]. A systematic review of 6 intervention studies has confirmed the portion size effect in 35 year old children, particularly in those $>4$ years [27], consistent with the idea that younger children have better self-regulation of appetite [26]. The effect has been seen most recently in a crossover RCT in 3-5 year old children, which found offering larger portions resulted in higher energy intake (10-15\% above calculated needs), with the greatest increases observed in more overweight children [28]. Larger servings (but notably not meal frequency), were also associated with greater weight gain in twins aged 2-5 years $(n=1939)$ [29], supporting the idea that providing practical advice on portion size could help prevent excessive weight gain [25].

\section{Physical Activity, Sedentary Behaviour and Sleep}


A lack of physical activity would be expected to contribute to a positive energy balance and hence risk of obesity. This concept is supported by a systematic review in 0-4 year old children, which reported that physical activity was associated with adiposity in 16 observational studies and 2 RCTs [30]. However, the same review found that 30 studies showed no association between physical activity and adiposity, and 4 even showed an unfavourable effect [30]. Moreover, a meta-analysis $(n=1100)$ of 3 cluster RCTs and 1 non-randomized intervention found no differences in BMI between intervention and control groups. Whilst physical activity has been consistently shown to have many health benefits in preschool children (e.g. for cardio-metabolic and psychological health, and physical, motor and cognitive development [30]) a causal link with obesity has not been established. For instance, a recent Cochrane systematic review found that interventions for obesity prevention that focused on physical activity alone were not effective in children aged 0-5 years [31]. Nonetheless, physical activity is an essential component of a healthy lifestyle and in the UK 3 hours/day at any intensity (including active and outdoor play) play is recommended for children aged 1-4 years.

Unsurprisingly, a low level of physical activity is associated with greater sedentary behaviour, defined as low energy sitting or reclining during waking hours [32]. Sedentary behaviour, such as a time spent in front of a screen, is postulated to be a risk factor for obesity independent of the time spent in moderate or vigorous physical activity. However, there is 
little evidence to support this widely accepted hypothesis in young children. For example, a systematic review of 60 studies that included 1 RCT and 13 longitudinal studies found no overall evidence for associations between objectively measured total sedentary time and health indicators (including adiposity) in children aged 0-4 years (as opposed to older children and adults) [32].

A third behaviour linked to adiposity in children is inadequate sleep. Two recent systematic reviews have confirmed that insufficient sleep is associated with obesity in preschool children $[33,34]$. A meta-analysis of 7 cohorts found that 'short' sleepers aged 0-3 years had a 40\% (95\%CI: 19 $65 \%$ ) greater risk of being overweight or obese $>1$ year later [34]. The mechanisms are unknown but a systematic review of 5 studies showed that poor sleep in preschool children was associated with poor diet quality and greater energy intake [35]. Although there is no casual evidence for a link between sleep and obesity, clearly, sleep has numerous health benefits for preschool children. However, many young children fail to get enough sleep (e.g. 10-13 hours per 24 hours for children aged 3-5 years recommended by the American Academy of Pediatrics). Improving sleep may therefore be a worthwhile target for public health interventions $[4,5]$.

Overall, although more physical activity, less sedentary behaviours and adequate sleep have huge health benefits in their own right, the scientific consensus is that the rise in childhood obesity is mostly driven by changes 
in food consumption rather than declines in physical activity [5]. Therefore, as recommended by the UK Chief Medical Officer [5], $80 \%$ of effort for obesity prevention should focus on improving the diet and $20 \%$ on increasing physical activity.

\section{Developmental Factors}

There is increasing evidence that developmental factors act in the early years to 'programme', or influence long-term risk of obesity, a concept known as the Developmental Origins of Health and Disease [9.20]. A systematic review of 282 prospective studies identified consistent association between several such factors acting in the first 1000 days (the period between conception and age 2 years) and later obesity including: maternal pre-pregnancy BMI, prenatal tobacco exposure, maternal excess gestational weight gain, high infant birthweight, and accelerated infant weight gain [9]. Fewer studies also supported a role for: gestational diabetes, child care attendance, low strength of maternal-infant relationship, low socio-economic status, curtailed infant sleep, inappropriate bottle use, introduction of solid food intake before age 4 months, and infant antibiotic exposure [9]. However, most studies are observational and, because of possible confounding, evidence of causality is limited. For instance, associations between gut microbiota (affected by antibiotic use) and obesity may be confounded because both exposure (microbiome) and outcome (obesity) are strongly affected by an unhealthy diet poor in fruit and vegetables. 
One developmental factor where there is more causal evidence is the impact of accelerated growth in infancy (usually as a result of higher protein intake) on later obesity. This hypothesis is supported by several RCTS [36] and seems to include effects of accelerated growth/higher protein intake in toddlers. For instance, a systematic review of 13 studies found that a higher protein intake between 6-36 months was associated with higher BMI later in childhood (pooled effect size 0.28 BMI z scores; 95\% CI: 0.20 to 0.35 ) $[17,37]$. The mechanisms for these effects are unknown but may involve programming of IGF-1 concentrations or effects on appetite regulation [36]. Since cow's milk is the major source of protein in young children, limiting cow's milk intake has been suggested as a possible intervention for obesity prevention (e.g. by ESPGHAN) [36].

Another development factor particular related to obesity in toddlers is programming of dietary habits and food preferences that can influence lifelong health. Analysis of children's diets by deriving a limited number of dietary patterns from a large variety of foods eaten using principal component analysis strongly supports this concept. These dietary patterns emerge in early life, are established by 3 years of age and are stable (or track) throughout childhood and into adult life (as reviewed [17]). As expected, unhealthy dietary patterns in children including toddlers are associated with a greater risk of later obesity [38]. However, in the absence 
of interventions studies, whether the diets of young children can be manipulated to ameliorate this risk is not known.

\section{Consequences of Childhood Obesity}

Many cohort studies, and more recently Mendelian randomisation studies, have established clear causal links between obesity in adults and adverse health consequences such cardiovascular disease (CVD), type 2 diabetes and cancer [13]. However, although the detrimental effects of obesity in older children and adults are well recognised, health care professionals and parents often fail to appreciate its harmful effect in toddlers and preschool children. Nevertheless there is strong evidence for adverse consequences of excess weight in toddlers for both short- and long-term health.

In the immediate or short-term, obesity in children is associated with problems similar to adults such as sleep apnoea, asthma, back and joint pain, and components of the metabolic syndrome such as high blood pressure, raised cholesterol, fatty liver disease and increased risk of type 2 diabetes [5]. In fact, in the UK there are 100 new cases of type 2 diabetes diagnosed in children each year [5]. Some complications of obesity are unique to children such as slipped femoral epiphyses and premature puberty [5]. Other immediate complications of obesity may be more marked in children such as psychological consequences, poor cognitive function and brain health, depression and bullying leading to low selfesteem and poor educational attainment [5]. All of these co-morbidities 
have a major impact on the individuals, parents, and health care services. However, of particular public health concern are the long-term consequences of childhood obesity on adult health. Obese children are $5 \mathrm{X}$ more likely to become obese adults [5] and several systematic reviews have shown associations between obesity in preschool children (0-6 years) and the metabolic syndrome in adults [39] and between obesity in older children ( $>7$ years) and adult type 2 diabetes, CVD, cancers and poorer [40]. Alarmingly, these effects seem to persist even in obese children who become slim adults.

\section{Overview}

Understanding the reasons behind the rapid increase in obesity in toddlers and young children over the last 50 years is a major challenge for scientists, health care professionals and policymakers worldwide. Although causality is often difficult to establish, many risk factors have been identified which act at both a societal and individual level. However, childhood obesity is complex and multifactorial, with each factor likely to make only a small contribution to overall risk. Interventions for prevention of obesity therefore need to target multiple risk factors and be implemented at society, community and family levels. Nonetheless, there is little doubt that investing in prevention of obesity in the early years will have huge health and economic benefits for populations, children and wider society [5].

The author declares no conflict of interest. 
(word count 3507) 


\section{References}

1. NCD Risk Factor Collaboration (NCD-RisC). Worldwide trends in bodymass index, underweight, overweight, and obesity from 1975 to 2016: a pooled analysis of 2416 population-based measurement studies in 128.9 million children, adolescents, and adults. Lancet 2017; 390: 2627-2642.

2. World Health Organisation (2018) Geneva. Obesity and Overweight Fact sheet: Available at:

https://www.who.int/en/news-room/fact-sheets/detail/obesity-andoverweight

3. Heymsfield SB, Wadden TA. Mechanisms, Pathophysiology, and Management of Obesity. N Engl J Med 2017; 376: 254-66.

4. World Health Organization (2016) Report of the Commission on Ending Childhood Obesity. Available at:

https://www.who.int/end-childhood-obesity/publications/echoreport/en/

5. Davies SC. Time to Solve Childhood Obesity, Department of Health Social Care 2019. Available at: https://www.gov.uk/government/organisations/department-of-healthand-social-care

6. Gardner DS, Hosking J, Metcalf BS, et al. Contribution of early weight gain to childhood overweight and metabolic health: a longitudinal study (EarlyBird 36). Pediatrics 2009; 123: e67-73. 
7. Geserick M, Vogel M, Gausche R, et al. Acceleration of BMI in Early Childhood and Risk of Sustained Obesity. N Engl J Med 2018; 379: 1303-1312.

8. Norman J, Kelly B, McMahon AT, et al. Sustained impact of energydense TV and online food advertising on children's dietary intake: a within-subject, randomised, crossover, counter-balanced trial. Int J Behav Nutr Phys Act 2018; 15: 37. doi: 10.1186/s12966-018-0672-6.

9. Woo Baidal JA, Locks LM, Cheng ER, et al. Risk Factors for Childhood Obesity in the First 1,000 Days: A Systematic Review. Am J Prev Med $2016 ; 50: 761-779$.

10. Ghobadi S, Hassanzadeh-Rostami Z, Salehi-Marzijarani M, et al. Association of eating while television viewing and overweight/obesity among children and adolescents: a systematic review and metaanalysis of observational studies. Obes Rev 2018; 19: 313-320.

11. Mazarello Paes V, Ong KK, Lakshman R. Factors influencing obesogenic dietary intake in young children (0-6 years): systematic review of qualitative evidence. BMJ Open 2015; 5: e007396.

12. Reilly JJ, Armstrong J, Dorosty AR, et al. Early life risk factors for obesity in childhood: Cohort study BMJ 2005; 330: 1357.

13. Goodarzi MO. Genetics of obesity: what genetic association studies have taught us about the biology of obesity and its complications. Lancet Diabetes Endocrinol 2018; 6: 223-236. 
14. Khera AV, Chaffin M, Wade $\mathrm{KH}$, et al. Polygenic Prediction of Weight and Obesity Trajectories from Birth to Adulthood. Cell 2019; 177: 587596.e9.

15. Elks CE, den Hoed M, Zhao JH, et al. Variability in the heritability of body mass index: a systematic review and meta-regression. Front Endocrinol (Lausanne) 2012; 3: 29. doi: 10.3389/fendo.2012.00029. eCollection

16. Müller MJ, Geisler, C, Blundell J, et al. The case of GWAS of obesity: does body weight control play by the rules? International Journal of Obesity 2018; 42: 1395-1405.

17. Lanigan J. Prevention of overweight and obesity in early life. Proc Nutr Soc. $2018 ; 77:$ 247-256.

18. Naude CE, Visser ME, Nguyen KA, et al. Effects of total fat intake on bodyweight in children. Cochrane Database Syst Rev 2018; 7: CD012960.

19. Hilger-Kolb J, Bosle C, Motoc I, et al. Associations between dietary factors and obesity-related biomarkers in healthy children and adolescents - a systematic review. Nutr J 2017; 16: 85. doi: 10.1186/s12937-017-0300-3.

20. Patro-Gołąb B, Zalewski BM, Kołodziej M, et al. Nutritional interventions or exposures in infants and children aged up to 3 years and their effects on subsequent risk of overweight, obesity and body fat: a systematic review of systematic reviews. Obes Rev 2016; 17: 1245-1257. 
21. Prynne CJ, Paul AA, Price GM, et al. Food and nutrient intake of a national sample of 4-year-old children in 1950: comparison with the 1990s. Public Health Nutr 1999; 2: 537-47.

22. Fidler Mis N, Braegger C, Bronsky J, et al. ESPGHAN Committee on Nutrition: Sugar in Infants, Children and Adolescents: A Position Paper of the European Society for Paediatric Gastroenterology, Hepatology and Nutrition Committee on Nutrition. Pediatr Gastroenterol Nutr 2017 Dec; 65: 681-696.

23. Frantsve-Hawley J, Bader JD, Welsh JA, et al. A systematic review of the association between consumption of sugar-containing beverages and excess weight gain among children under age 12. J Public Health Dent 2017; 77 Suppl 1: S43-S66.

24. Vercammen KA, Frelier JM, Lowery CM, et al. A systematic review of strategies to reduce sugar-sweetened beverage consumption among 0 year to 5-year olds. Obes Rev 2018; 19: 1504-1524.

25. Hetherington MM, Blundell-Birtill P, Caton SJ, et al. Understanding the science of portion control and the art of downsizing. Proc Nutr Soc 2018; 77: 347-355.

26. Rolls BJ, Engell D, Birch LL. Serving portion size influences 5-year-old but not 3-year-old children's food intakes. J Am Diet Assoc $2000 ; 100$ : $232-4$

27. Small $L$, Lane $H$, Vaughan $L$, et al. A systematic review of the evidence: the effects of portion size manipulation with children and portion 
education/training interventions on dietary intake with adults. Worldviews Evid Based Nurs 2013; 10: 69-81.

28. Smethers AD, Roe LS, Sanchez CE, et al. Portion size has sustained effects over 5 days in preschool children: a randomized trial. Am J Clin Nutr 2019; 109: 1361-1372.

29. Syrad $\mathrm{H}$, Llewellyn $\mathrm{CH}$, Johnson $\mathrm{L}$, et al. Meal size is a critical driver of weight gain in early childhood. Sci Rep 2016; 6: 28368.

30. Carson V, Lee EY, Hewitt $L$, et al. Systematic review of the relationships between physical activity and health indicators in the early years (0-4 years). BMC Public Health 2017; 17 (Suppl 5): 854.

31. Brown $T$, Moore $T H$, Hooper $L$, et al. Interventions for preventing obesity in children. Cochrane Database Syst Rev 2019; 7: CD001871.

32. Poitras VJ, Gray CE, Janssen X, et al. Systematic review of the relationships between sedentary behaviour and health indicators in the early years (0-4 years). BMC Public Health 2017; 17(Suppl 5): 868.

33. Chaput JP, Gray CE, Poitras VJ, et al. Systematic review of the relationships between sleep duration and health indicators in the early years (0-4 years). BMC Public Health. 2017; 17 (Suppl 5): 855.

34. Miller MA, Kruisbrink M, Wallace J, et al. Sleep duration and incidence of obesity in infants, children, and adolescents: a systematic review and meta-analysis of prospective studies. Sleep 2018; 41(4).

35. Ward AL, Reynolds AN, Kuroko S, et al. Bidirectional associations between sleep and dietary intake in 0-5 year old children: A systematic review with evidence mapping. Sleep Med Rev 2019; 49: 101231. 
36. Singhal A. The role of infant nutrition in the global epidemic of noncommunicable disease. Proc Nutr Soc 2016; 75: 162-168.

37. Lanigan J, Adeboye A, Northstone $\mathrm{K}$, et al. Nutrition in preschool chidlren and later risk of obesity: a systematic review and metaanalysis.J Pediatric Gastroenterol Nutr 2017; 62: Suppl 1. 691.

38. Liberali R, Kupek E, de Assis MAA. Dietary Patterns and Childhood Obesity Risk: A Systematic Review. Child Obes 2019; 19

39. Kim J, Lee I, Lim S. Overweight or obesity in children aged 0 to 6 and the risk of adult metabolic syndrome: A systematic review and metaanalysis. J Clin Nurs 2017; 26: 3869-3880.

40. Llewellyn A, Simmonds M, Owen CG, et al. Childhood obesity as a predictor of morbidity in adulthood: a systematic review and metaanalysis. Obes Rev 2016; 17: 56-67. 